\title{
Methods of aggregation of expert opinions in the framework of intelligent products
}

\author{
S. Kubler ${ }^{* *}$ W. Derigent ${ }^{*}$ A. Voisin ${ }^{*}$ K. Främling ${ }^{* *}$ \\ A. Thomas* \\ ${ }^{*}$ Centre de Recherche en Automatique de Nancy, Université de \\ Lorraine, CNRS UMR 7039 \\ Campus Sciences, BP 70239, F-54506 Vandœuvre-lès-Nancy, France \\ ** Aalto University, School of Science and Technology, Finland, \\ (e-mail:s.kubler@univ-lorraine.fr)
}

\begin{abstract}
Intelligent products may be considered as information vectors, conveying their own information from a phase of its life cycle to another. Considering that such a product could be made with "communicating material", in previous work, a data dissemination process has been developed with the aim of selecting information from a database, that should be stored on the product according to the current stage of its life cycle. This selection is based on an indicator of relevance which depends on a multitude of criteria, whose weights are evaluated by a group of experts. The set of expert opinions must be formalized in accordance with a mathematical theory and then, synthesized by a suitable aggregation method. In this framework, three aggregation methods are introduced and compared. This comparison is carried out based on the scenario of data dissemination when using intelligent products. Results enable to assess the goodness of fit of the three aggregation methods.
\end{abstract}

Keywords: Intelligent product, Fuzzy set theory, Expert opinion, Aggregation, Fusion

\section{INTRODUCTION}

Intelligent Products have been introduced as a concept for making physical products become autonomous actors that can optimize their operations, usage and other behavior in order to fulfill their "mission", which may depend on their current context (Meyer et al. (2009)). Products, as well as their parts and materials, have different life cycles through which they usually move in numerous companies. Technical, semantic and organizational interoperability between these companies is not always ensured, thereby contributing to information loss. Considering the product as an information vector (i.e. to which information can be associated) should contribute to improved interoperability and sustainability throughout its life cycle (Wong et al. (2002)). However, there are still some open questions like how information should be gathered, stored and distributed over the Product Life Cycle - PLC (McFarlane et al. (2012)). To address this issue, a data dissemination process has been developed in previous work with the aim of allocating information between databases and intelligent products (Kubler et al. (2012a,c)). More concretely, this process selects data "appropriate" to the expected situation from the database system (i.e., the data that should be stored on the product) thanks to an indicator of relevance which gives the degree to which information might be useful for the subsequent actors (e.g. manufacturers, users, recyclers).

In our process, the indicator of relevance is dependent upon several criteria and involves numerous experts during the assessment phase. Experts are solicited, for instance, to compare criteria with each other. When several experts are questioned on such parameters, the set of opinions must be formalized in accordance with a mathematical theory and then, synthesized by a suitable aggregation method (Destercke et al. (2006)). The objective of aggregation is to combine individual sources of information into an overall one in a proper way, so that the final result of aggregation can take into account all the individual contributions (Dubois and Prade (1985)). Conflicts among experts may therefore occur and an effective method of aggregation must be defined to handle this problem. The main purpose of this paper is not to detail the exact aggregation process of expert opinions but to compare three different aggregation methods based on the scenario of data dissemination.

Section 2 introduces the two main theories used in the literature to develop aggregation methods of expert opinions. Section 3 first briefly outlines the data dissemination process (in which product-related data is allocated between a database and an intelligent product) and then, details the three methods of aggregation that should be compared. Section 4 presents the process implemented to compare these three methods. Finally, results are provided in section 5 , through which advantages and limitations of each method are pointed out.

\section{AGGREGATION METHODS VIA UNCERTAINTY AND PROBABILITY THEORIES}

It is common practice to solicit experts for assessing model parameters that are poorly known due to the lack of data. As mentioned previously, the set of expert opinions must be formalized in accordance with a mathematical theory 
and then, synthesized by a suitable aggregation method. When conflicts occur among experts, an effective way to manage the conflict consists in assigning weights to experts (i.e. by assessing their quality). Such operations are usually carried out in the framework of probability theory (Cooke (1991)). The probabilistic approach, although it has proved its worth, is ill-suited to model vague opinions and limits the choice of aggregation methods as argued by Destercke et al. (2006). Uncertainty theories such as fuzzy set theory enable to overcome these limits. Recent years have witnessed many proposals in fuzzy set theory (Lee (2002); Chiclana et al. (2012)).

Let us consider a parameter $v$ on a domain $\mathcal{X}$ to be estimated. Two experts $e_{1}$ and $e_{2}$ give their opinion $e_{1}(v)=A$ and $e_{2}(v)=B$ with $A, B \subseteq \mathcal{X}$. Let $\bowtie$ be the aggregation operator. According to Destercke et al. (2006), an aggregation method can be characterized by one of the three following behaviors:

- conjunctive behavior: $e_{1}(v) \bowtie e_{2}(v) \subseteq A \cap B$. The result is more accurate than the expert's one and can be used when the opinions are not conflicting (i.e. $\left.e_{1}(v) \bowtie e_{2}(v) \notin \emptyset\right)$,

- disjunctive behavior: $e_{1}(v) \bowtie e_{2}(v) \supseteq A \cup B$. The result is less accurate than previously but all opinions are taken into account. In fact, the disjunction represents the case where the modeler does not want to choose among the expert opinions which might be conflicting,

- "counting" behavior: the result corresponds to a statistical view of the opinions. A counting behavior could actually be defined at the interface between the disjunctive and the conjunctive ones.

In practice, it makes sense to adapt the aggregation method according to the context. Indeed, the accuracy and the nature of the aggregated result depends on both the presence of conflicts and the available knowledge on the expert quality. In this paper, the scenario of data dissemination (described in section 3.1) involves experts whose points of view are all legitimate and must be taken into account. Accordingly, a conjunctive behavior for the aggregation is not suitable and should rather be disjunctive, or maybe even counting. In this sense, two methods of aggregation based on the fuzzy set theory are considered in this paper to achieve the disjunctive behavior, one proposed by Chen (2005) ${ }^{1}$ and another developed by Kubler et al. (2012c). The third method, which tends to characterize a counting behavior, is based on the probability theory. It would be interesting to compare the three approaches and, by the way, both behaviors. These three methods are introduced in section 3 .

\section{THE EXPLORED AGGREGATION METHODS}

Section 3.1 outlines the main steps of the data dissemination process and gives insight into the scenario on which it is applied. Then, section 3.2 introduces the three methods of aggregation that are compared in this paper.

\footnotetext{
1 Their method has also been instantiated considering the scenario of data dissemination in Kubler et al. (2012a).
}

\subsection{Data dissemination process}

As mentioned previously, the data dissemination process developed in Kubler et al. (2012a,c) aims at selecting data appropriate to the expected situation. The selected data are thus stored on the intelligent product. This process is performed at different stages in the PLC. Fig. 1 gives insight into a PLC and highlights two different points where the product is written (i.e. when the data dissemination process is executed: $c f$. solid arrow) and one point where the product is read (i.e. when data carried by the product are retrieved and then, reinjected in the database: $c f$. dashed arrow).

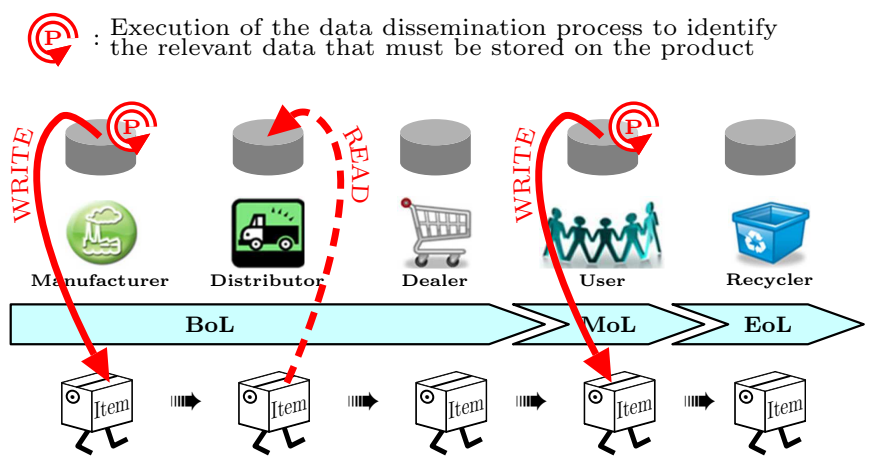

Fig. 1. Data dissemination process over the PLC

The process uses the Logical Data Model (LDM). Fig. 2 gives insight into a part of a LDM, where one entity corresponds to a relational table as shown with the entity/table Material. The attributes listed in each entity correspond to the table columns and each row is referred to as a tuple. For instance, Material has 3 attributes and 4 tuples. One data item corresponds to one table cell. The data dissemination process ultimately assesses the relevance of all product-related data items, from all tables $^{2}$. The higher the relevance value, the higher the necessity that this data item should be stored on the product. For instance, the three data items composing the tuple 3 in Fig. 2, noted $\mathrm{T}_{\mathrm{Mat}\{3,1\}}, \mathrm{T}_{\operatorname{Mat}\{3,2\}}$ and $\mathrm{T}_{\mathrm{Mat}\{3,3\}}$, are somehow in relation with the product when the others are not (represented by the hatched background). Accordingly, only these 3 data items are assessed and, in this example, $\mathrm{T}_{\mathrm{Mat}\{3,3\}}$ is the most relevant with a value of 0.40 .

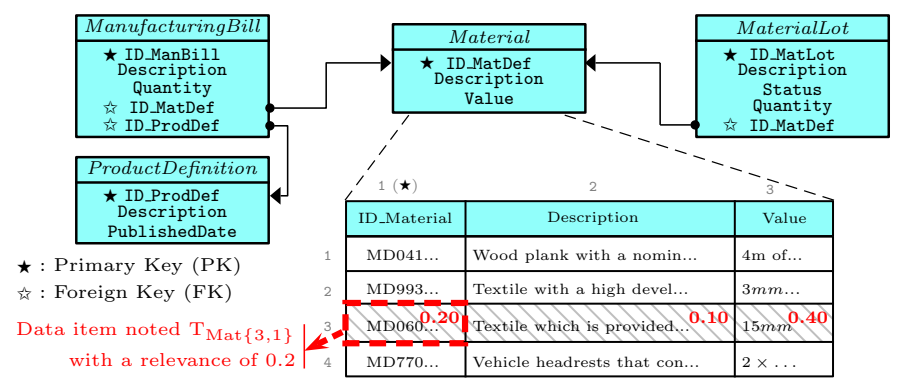

Fig. 2. Logical Data Model (LDM) \& relational table

The relevance value is computed thanks to a multi-criteria decision making (MCDM) method, namely the Analytical

\footnotetext{
2 An algorithm is developed in Kubler et al. (2012b) to identify all
} product related data items from the database. 

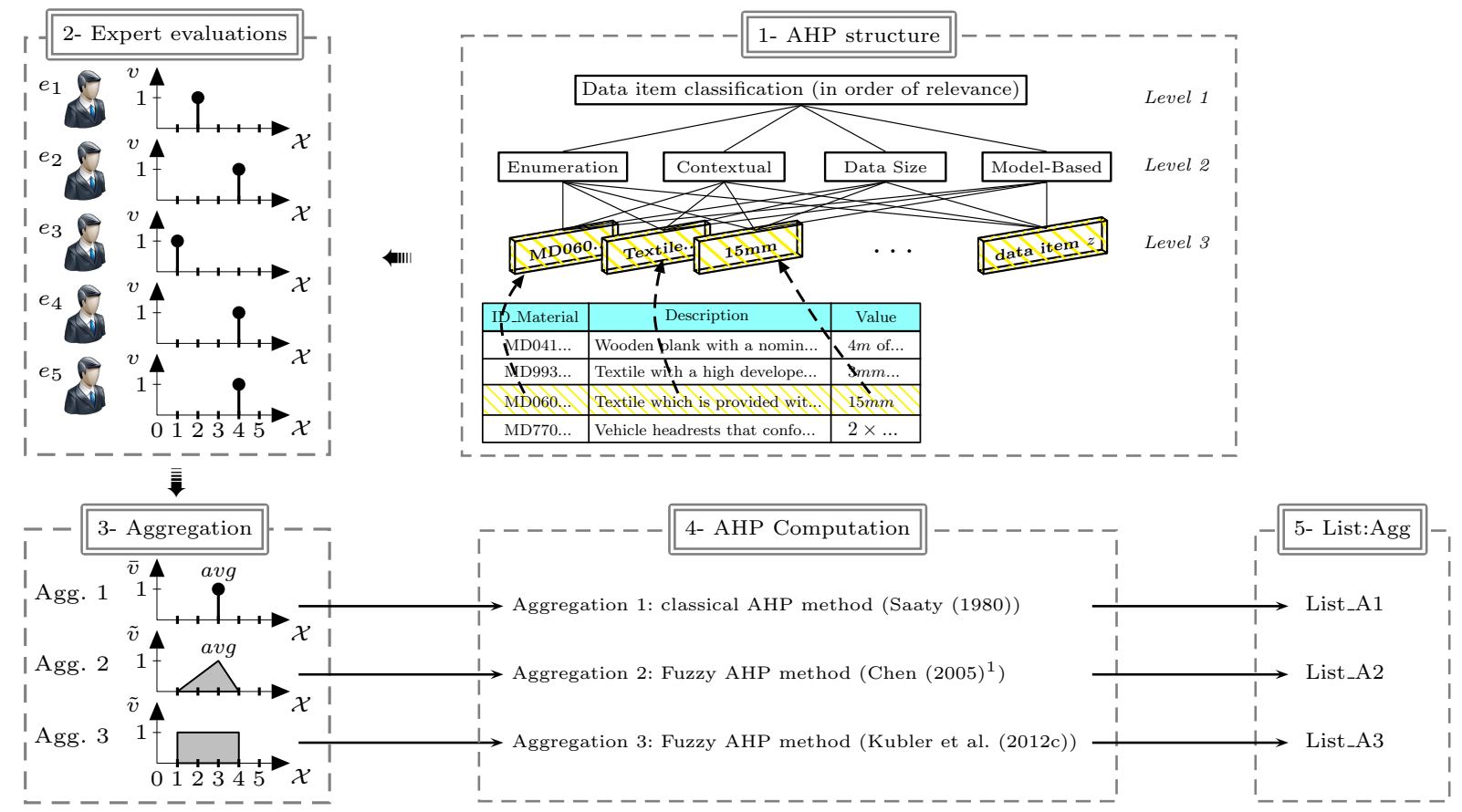

Fig. 3. Illustration of the three methods of aggregation of expert opinions based on the scenario of data dissemination

Hierarchy Process (AHP). This method, originally proposed by Saaty (1980), has the advantage to organize the critical aspects of the problem in a hierarchical structure, thus facilitating the decision making process. Our MCDM problem is broken down into the hierarchical structure depicted in Fig. 3 ( $c f$. frame named: 1-AHP structure). The alternatives are the product-related data items ( $c f$. level 3) that must be assessed and ranked in term of relevancy $(c f$. level 1). Four criteria are defined at level 2: Enumeration, Contextual, Data Size and Model-Based which are respectively abbreviated $\mathrm{C}_{e}, \mathrm{C}_{c}, \mathrm{C}_{s}, \mathrm{C}_{m}$ :

- $\mathrm{C}_{e}$ : through this criterion, experts may enumerate table attributes they judge important to store on the product,

- $\mathrm{C}_{c}$ : this criterion allows to evaluate more globally than with $\mathrm{C}_{e}$ the information that should be selected. Indeed experts may not be aware of all the data needed by the downstream actors of the PLC. In this second criterion, experts assess the importance of several families of information according to the location of the product in the PLC (e.g. importance of accounting information, manufacturing information),

- $\mathrm{C}_{s}$ : this criterion favors the storage of data items on the product according to their size. Since products are often memory-constrained, the data relevance in our approach decreases when the data size increases,

- $\mathrm{C}_{m}$ : this criterion is based on the relationships implied through the LDM. The shorter the distance between tables, the higher the data correlation and therefore the data relevance.

In our AHP process, several experts express their opinion regarding many parameters (parameters in $\mathrm{C}_{e}$, in $\mathrm{C}_{c}$, pairwise comparisons between criteria,... ). As stated previously, the objective of this paper is not to detail how experts perform these evaluations ${ }^{3}$ but to compare the

3 This is available in Kubler et al. (2012a) and Kubler et al. (2012c). methods which are used to aggregate the different points of view of experts on a same parameter $v$. The frame entitled "2-Expert evaluations" in Fig. 3 provides the evaluations performed by 5 experts on a parameter $v$. Three methods of aggregation are thus proposed and described in section 3.2 .

\subsection{Description of the three methods of aggregation}

In our case, experts provide crisp evaluations as depicted in Fig. 3 ("2-Expert evaluations"). It is therefore necessary to find the most suitable aggregation method. As stated in section 2, the intended behavior of the aggregation method should rather be disjunctive or still, counting than conjunctive. For that purpose, one aggregation method based on the probability theory (to get a counting behavior) and two methods based on the fuzzy set theory (to get a disjunctive behavior) are compared. In order to detail each method, let us consider the evaluations of parameter $v$ performed by five experts on a domain $\mathcal{X}=\{1,2,3,4,5\}$ as follows: $e_{1}(v)=2, e_{2}(v)=4, e_{3}(v)=1, e_{4}(v)=4$ and $e_{5}(v)=4$ (see Fig. 3). The three aggregation methods are:

- method 1: the aggregation operator $\bowtie$ is the average of the expert assessments as illustrated in Fig. 3. The average opinion $\bar{v}$ is thus equal to 3 $\left(=\frac{e_{1}(v)+e_{2}(v)+e_{3}(v)+e_{4}(v)+e_{5}(v)}{5}\right)$,

- method 2: the aggregation operator $\bowtie$ consists in integrating the expert opinions through a triangular fuzzy set $\tilde{v}$. The kernel of this set is equal to the average evaluation and then, uncertainty is added in a decreasing way from this value (i.e. until reaching the minimal and maximal opinions formulated by the experts) as depicted in Fig. 3. This representation tends to mean that the " best/correct" evaluation is considered as the average evaluation and that uncertainty arise from the set of evaluations. 
- method 3: the aggregation operator $\bowtie$ consists in integrating all expert opinions through an uniform fuzzy set, where the lower and upper limits are respectively the lower and upper opinions as depicted in Fig. 3. In the resulting fuzzy set, all opinions are of equal importance unlike the two previous aggregation methods. Indeed, the two previous modeling imply, ultimately (i.e. after applying the aggregation process), that opinions are no longer equal since the average opinion is considered as the most "correct" evaluation. Considering the aggregation method 3 , one may add uncertainty by extending the limits of the uniform fuzzy set. However, in order not to complicate the study, such possibility is not presented in this paper ( $c f$. Kubler et al. (2012c)).

Readers who need further information about the aggregation methods 1, 2 and 3 can respectively consult Saaty (1980); Kubler et al. (2012a,c). In these articles, the AHP computation steps are described as indicated by the frame "4-AHP Computation" in Fig. 3. At the end of the process, a list of data items ordered from the most relevant to the lowest is produced by each aggregation method, noted respectively List_A1, List_A2 and List_A3 in Fig. 3 ( $c f$. frame "5-List:Agg"). The next section details the comparison process that is implemented to assess the goodness of fit of the three aggregation methods.

\section{COMPARISON PROCESS}

The aim of the comparison process is to assess each aggregation method, i.e to determine the satisfaction of each expert regarding the aggregation result. Each method of aggregation $i$ generates a list named List_A $i$. In order to assess the performance of the aggregation method, the idea is to compare the similarity between the aggregated list (i.e. List_A $i$ ) with the list resulting of the specification of a single expert $k$ when using the method $i$ (list noted List_A $\left.i: e_{k}\right)$. Fig. 4 gives the example where only the opinion provided by expert 4 about the parameter $v$ is used by the aggregation method $i$ ( $c f$. frame 2,3 and 4 ), thus generating three lists: List_A1: $e_{4}$, List_A2: $e_{4}$ and List_A $3: e_{4}$. Ultimately, if the list resulting from the single expert $k$ is entirely similar to the list resulting of the aggregation of all experts, that means that the aggregation method $i$ entirely satisfies the expert $k$ (i.e. the intended goal). The Jaccard similarity coefficient (Tan et al. (2006)) could be used to compare two distinct lists $A$ and $B$. It is defined as in equation 1.

$$
J(A, B)=\frac{|A \cap B|}{|A \cup B|}=\frac{|A \cap B|}{z}
$$

$J$ measures the similarity between both lists and is defined as the size of the intersection divided by the size of the union of the lists. Thus, it helps to determine the proportion of common data items in both lists. It goes from 0 (no common data items) to 1 (identical lists). Let $A, B$ and $B^{\prime}$ be three lists of data items in which each data item get a final rank as illustrated in Fig. 5 (rank related to their relevance to be stored on the product). In this example, two Jaccard similarity coefficients $J(A, B)$ and $J\left(A, B^{\prime}\right)$ are computed. Both coefficients are equal because the union of $|A \cap B|=\{1,2,3\}$ and $\left|A \cap B^{\prime}\right|=\{1,2,3\}$ give the same cardinality, namely 3 .

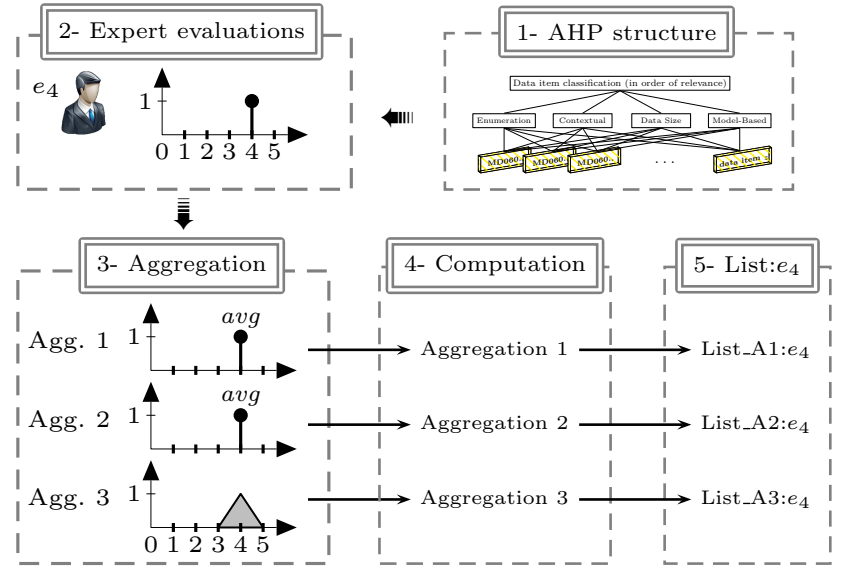

Fig. 4. Aggregation methods considering a single expert

\begin{tabular}{|c|c|c|c|c|c|c|}
\hline \multicolumn{2}{|c|}{$A$} & \multicolumn{2}{|c|}{$B$} & \multicolumn{2}{|c|}{$B^{\prime}$} & \multirow{2}{*}{$J(A, B)=\frac{|A \cap B|}{z}$} \\
\hline $\mathrm{n}^{\circ} \mathrm{DI}$ & rank & $\mathrm{n}^{\circ} \mathrm{DI}$ & rank & $\mathrm{n}^{\circ} \mathrm{DI}$ & rank & \\
\hline D1 & 1 & D1 & 1 & D1 & 7 & \\
\hline D2 & 2 & D2 & 2 & D2 & 6 & -5 \\
\hline D3 & 3 & D3 & 3 & D3 & 1 & $D^{\prime}\left(D^{\prime}\right) \quad|A \cap B|$ \\
\hline D4 & 4 & D4 & 6 & D4 & 2 & $J\left(A, B^{\prime}\right)=\frac{|A| D \mid}{z}$ \\
\hline D5 & 5 & D5 & 7 & D5 & 3 & $=\frac{3}{5}$ \\
\hline
\end{tabular}

Fig. 5. Computation of Jaccard similarity coefficients

In our data dissemination process, data items extracted from the database are ordered by relevance, the $1^{\text {st }}$ data item being the most important. During writing operations, data items are thus written from rank 1 to $z$, until no memory is available. It is thus interesting to define a similarity coefficient that would take the rank into consideration. Indeed, more important is our attention on similarities at the beginning of the lists than at the end since data items are ordered in term of relevance. To do so, let us define $L_{p}$ as the sublist of $L$ composed of data items from rank 1 to $p(p \leq z)$. It is then possible to compute a progressive similarity coefficient $J_{p}(A, B)$ as in equation 2 , used to know the similarity evolution between both lists. Fig. 6 details the results obtained when computing the evolution of the Jaccard progressive coefficients $J_{p}(A, B)$ and $J_{p}\left(A, B^{\prime}\right)$, for all $p=\{1,2, \ldots, 5\}$ in this example.

$$
J_{p}(A, B)=J\left(A_{p}, B_{p}\right)
$$

$$
\begin{aligned}
& J_{1}(A, B)=\frac{\left|A_{1} \cap B_{1}\right|}{1}=1 \\
& J_{2}(A, B)=\frac{\left|A_{2} \cap B_{2}\right|}{2}=1 \\
& J_{3}(A, B)=\frac{\left|A_{3} \cap B_{3}\right|}{3}=1 \\
& J_{4}(A, B)=\frac{\left|A_{4} \cap B_{4}\right|}{4}=0.75 \\
& J_{5}(A, B)=\frac{\left|A_{5} \cap B_{5}\right|}{5}=0.6
\end{aligned}
$$

$$
\begin{aligned}
& J_{1}\left(A, B^{\prime}\right)=\frac{\left|A_{1} \cap B_{1}^{\prime}\right|}{1}=0 \\
& J_{2}\left(A, B^{\prime}\right)=\frac{\left|A_{2} \cap B_{2}^{\prime}\right|}{2}=0 \\
& J_{3}\left(A, B^{\prime}\right)=\frac{\left|A_{3} \cap B_{3}^{\prime}\right|}{3}=0.33 \\
& J_{4}\left(A, B^{\prime}\right)=\frac{\left|A_{4} \cap B_{4}^{\prime}\right|}{4}=0.5 \\
& J_{5}\left(A, B^{\prime}\right)=\frac{\left|A_{5} \cap B_{5}^{\prime}\right|}{5}=0.6
\end{aligned}
$$

Fig. 6. Computation of Jaccard progressive coefficients

To determine the similarity between a list produced by an aggregation method $i$ (List_A $i$ ) with the list resulting of the specification of a single expert $k$ when using the method $i$ (List_A $i: e_{k}$ ), the progressive Jaccard coefficients are thus computed. As mentioned, the evolution is an indicator of the expert satisfaction ${ }^{4}$. Fig. 7 shows that, for an aggregation method $i, m$ progressive Jaccard coefficients (curves) are then obtained, with $m$ the number

\footnotetext{
4 If the aggregation list is identical to the expert's one, the expert is considered as entirely satisfied. This is a strong assumption since the expert's list is considered as a correct expression of his opinion.
} 
of experts (graph entitled similarity with each expert). In this example, 5 different comparisons of similarity are performed since 5 experts are considered (each list being composed of $z$ data items).

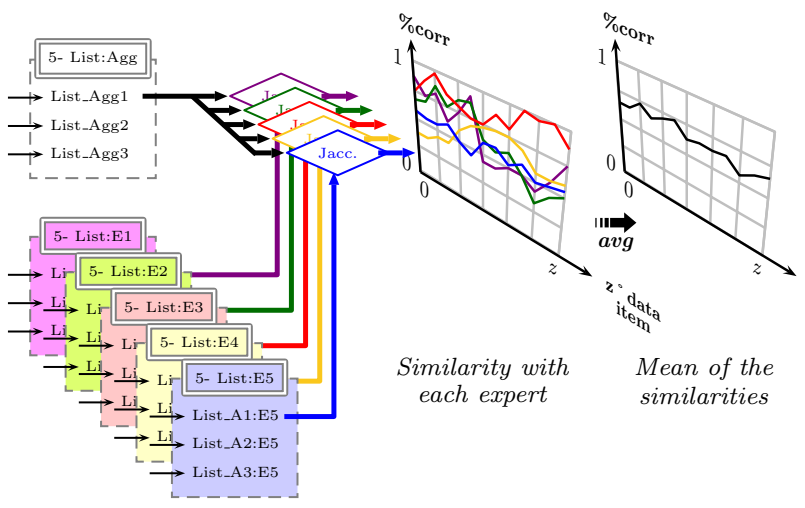

Fig. 7. Comparison process based on correlations

Nonetheless, the main objective of an aggregation method when a disjunctive behavior is desired consists in finding the best consensus among the experts (i.e. to find an aggregated list which is the most similar to all the expert's list). Accordingly, the mean progressive Jaccard coefficients is computed via equation 3 for each aggregation method $i$, which is noted $J_{p}$ (List:A $\left.i\right)$. The second graph in Fig. 7, entitled mean of similarities, shows the evolution of the mean progressive Jaccard coefficients related to the aggregation method 1 , which is obtained from the 5 similarity measures of the first graph (i.e. similarity with each expert).

$$
J_{p}(\text { List:A } i)=\frac{\sum_{k=1}^{m} J_{p}\left(\text { List:A } i, \text { List }: \mathrm{A} i: e_{k}\right)}{m}
$$

\section{COMPARISON RESULTS}

In our study and considering the scenario of data dissemination process, 5 experts noted $e_{1}, e_{2}, e_{3}, e_{4}$ and $e_{5}$ have performed evaluations regarding the criteria Enumeration, Contextual, Data size and Model-based. These evaluations are not described in detail but will be commented all along this section (i.e. when explaining the results). Two scenarii are defined based on these evaluations:

(1) Importance of $C_{e}$ : the Enumeration criterion is strongly favored over the other criteria ${ }^{5}$,

(2) Equal-importance: the four criteria are of equal importance,

Among the 5 experts, $e_{5}$ enumerates information that is not at all enumerated by the 4 other experts. As mentioned previously, this opinion is as legitimate as the 4 others. Accordingly, it would be interesting to analyze whether the aggregation methods tends to satisfy or not each expert. This is particularly interesting in scenario (1), when $\mathrm{C}_{e}$ is judged as the most important criterion. By applying the comparison process described in section 4 , the Jaccard progressive coefficients between the list of each aggregation method $i$ (List_A $\forall i=\{1,2,3\})$ and the list of each expert $k$ (List_A $i: e_{k} \forall k=\{1,2,3,4,5\}$ ) are computed and displayed in Fig. 8 for scenarii (1) and (2). Regarding the first scenario Fig. 8(a), it can

\footnotetext{
5 In AHP, this specification is made when performing the pairwise comparisons between criteria (i.e. $\mathrm{C}_{e}, \mathrm{C}_{c}, \mathrm{C}_{s}$ and $\mathrm{C}_{m}$ ).
}

be observed that in the three aggregation methods it is difficult to satisfy $e_{5}$. Indeed, the curves related of the Jaccard progressive coefficients of $e_{5}$ always remain far below the 4 other curves and, especially until reaching the $50^{\text {th }}$ data item. This can be explained by the fact that $e_{5}$ has an isolated point of view and it is not that simple to satisfy every expert. Nonetheless, among the three aggregation methods, method 3 satisfies the most $e_{5}$. Indeed, methods 1 and 2 have a similarity of 0 approximatively between the data items $n^{\circ} 1$ to 30 , while method 3 has a similarity of 0.3 approximatively. The consequence is that the satisfaction rating of the 4 other experts in method 3 is a bit lesser than in methods 1 and 2, but nothing significant. Indeed, if we look at the impact on the mean progressive Jaccard coefficients given in Fig. 9(a), it can be noted that, in average, the three methods of aggregation satisfy in an equivalent manner the experts. This confirms the fact that method 3 better meets the disjunctive behavior than the two other methods since it tries not to neglect/to leave out opinions.

Let us focus now on scenario (2) whose criteria are of equal importance. This time, considering the results of Fig. 8(b), it can be concluded that no expert has been left out or treated unfairly. Indeed, unlike scenario (1), no curves are detached from others, meaning that each aggregation method equally satisfies the experts. It should nevertheless be noted that, in Fig. 9(b), method 3 better satisfies the experts in average. This is especially true in view of the beginning of the lists (until the $50^{\text {th }}$ data item) on which our attention is mainly focused.

More generally, we can conclude that the three aggregation methods have globally a similar behavior. Indeed, the curves related to the mean Jaccard progressive coefficients of methods 1, 2 and 3 are almost always overlapping, either in scenario (1) or (2) (see Fig. 9).

\section{CONCLUSION}

In this paper, three aggregation methods of expert opinions are compared, based on a scenario of data dissemination using intelligent products. Experiments are conducted based on two scenarii, the first one favoring a given criterion over others and, the second one, considering criteria as of equal importance. Results showed that these three methods have globally the same behavior, which means that introducing uncertainty does not impact significantly the expert satisfaction. However, this study has shown that the best disjunctive behavior is obtained with the fuzzy aggregation operator developed by Kubler et al. (2012c). As a conclusion, this study seems to prove that fuzzy aggregation operators suit best to our context, compared to classical counting methods. These preliminary results should further be validated by a wider study including additional experiments. Ideally, it will lead to an adaptive method selecting the best suited aggregation method, depending on the criteria importance.

\section{ACKNOWLEDGEMENTS}

The authors gratefully acknowledge the financial support of the CPER 2007-2013 (Competitiveness Fibre Cluster), through regional (Région Lorraine), national (DRRT and FNADT) and European (FEDER) funds. 

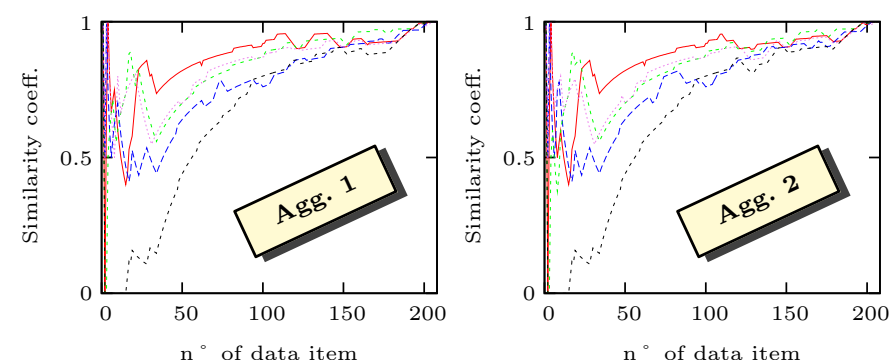

$\mathrm{n}^{\circ}$ of data item

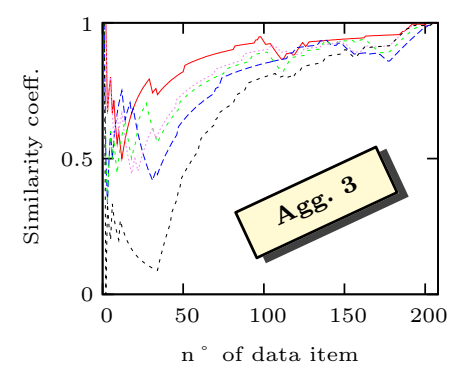

(a) Jaccard progressive coefficients in scenario (1)
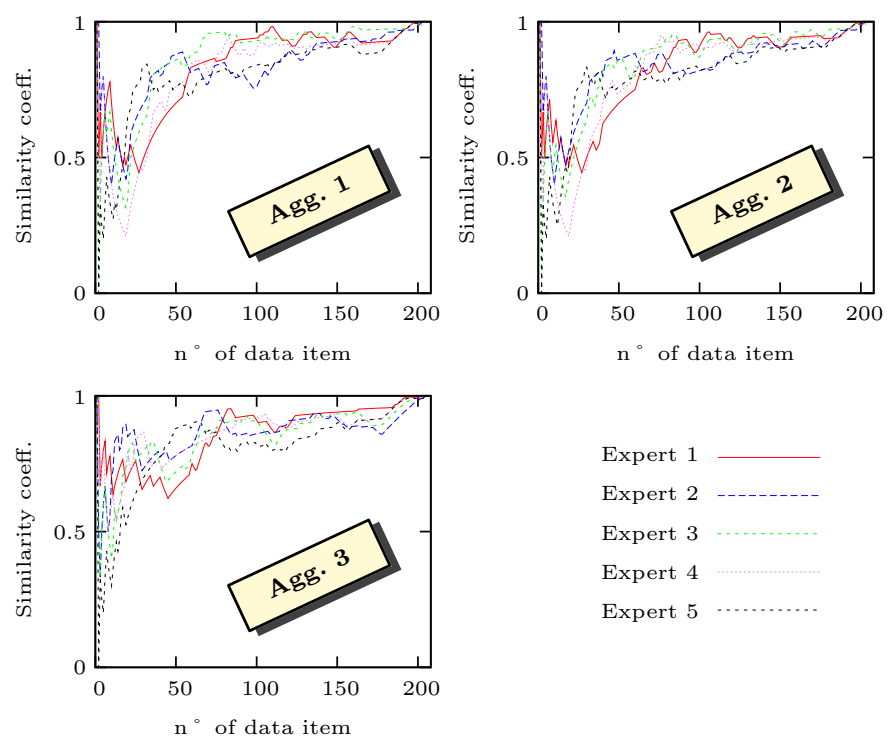

(b) Jaccard progressive coefficients in scenario (2)

Fig. 8. Computation of Jaccard progressive coefficients for each expert with respect to each aggregation method

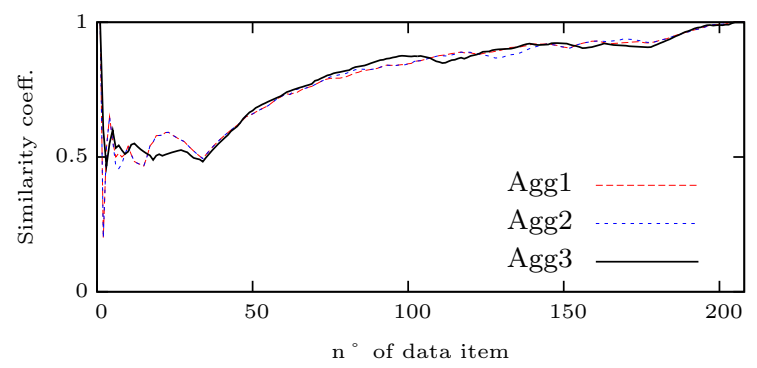

(a) Mean Jaccard progressive coefficients in scenario (1)

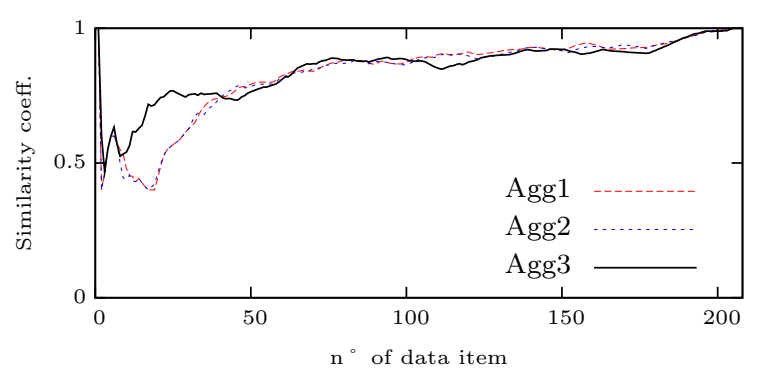

(b) Mean Jaccard progressive coefficients in scenario (2)

Fig. 9. Computation of the mean Jaccard progressive coefficients for each aggregation method

\section{REFERENCES}

Chen, H. (2005). A research based on fuzzy AHP for multicriteria supplier selection in supply chain. Master's thesis, University of Science and Technology of Taiwan.

Chiclana, F., Tapia García, J., del Moral, M., and HerreraViedma, E. (2012). A statistical comparative study of different similarity measures of consensus in group decision making. Information Sciences, 221, 110-123.

Cooke, R. (1991). Experts in uncertainty: opinion and subjective probability in science. Oxford University Press, USA.

Destercke, S., Dubois, D., and Chojnacki, E. (2006). Aggregation of expert opinions and uncertainty theories. In Rencontres Francophones sur la Logique Floue et ses Applications, 295-302.

Dubois, D. and Prade, H. (1985). A review of fuzzy set aggregation connectives. Information sciences, 36(1), 85-121.

Kubler, S., Derigent, W., Thomas, A., and Rondeau, É. (2012a). Embedding information on communicating materials from context-sensitive information analysis based on fuzzy ahp theory. In International Conference on Green Computing and Communications, 240-243.

Kubler, S., Derigent, W., Thomas, A., and Rondeau, É. (2012b). Information dissemination process for contextaware products. In 14th Symposium of Information
Control Problems in Manufacturing.

Kubler, S., Voisin, A., Derigent, W., Rondeau, É., and Thomas, A. (2012c). A fuzzy analytic hierarchy process for group decision making: application for embedding information on communicating materials. In IEEE International Conference on Communications, Computing and Control Applications, 270-276.

Lee, H. (2002). Optimal consensus of fuzzy opinions under group decision making environment. Fuzzy Sets and Systems, 132(3), 303-315.

McFarlane, D., Giannikas, V., Wong, A.C., and Harrison, M. (2012). Intelligent products in the supply chain 10 years on. In 14 th Symposium of Information Control Problems in Manufacturing.

Meyer, G., Främling, K., and Holmström, J. (2009). Intelligent products: A survey. Computers in Industry, 60(3), $137-148$.

Saaty, T. (1980). The Analytic Hierarchy Process. New York: McGraw-Hill.

Tan, P.N., Steinbach, M., and Kumar, V. (2006). Introduction to data mining. Addison-Wesley Longman Publishing Co., Inc.

Wong, C.Y., Mcfarlane, D., Zaharudin, A.A., and Agarwal, V. (2002). The Intelligent Product Driven Supply Chain. In International Conference on Systems, Man and Cybernetics, 4-6. 